\title{
Activités
}

18-2 | 2021

Espace et temps de l'activité / Travail, Territoire et politique

\section{"Si on ne me fournit pas mes engins, après je peux plus rien faire moi" : les apports de l'analyse de l'activité à la compréhension des processus d'engagement et de désengagement au travail}

"If you don't give me the required equipment, I won't be able to do anything": the contribution of the analysis of activity to the understanding of commitment and disengagement processes at work"

\section{Amélie Sandoval et Katia Kostulski}

\section{OpenEdition}

Journals

Édition électronique

URL : https://journals.openedition.org/activites/6835

DOI : 10.4000/activites.6835

ISSN : $1765-2723$

Éditeur

ARPACT - Association Recherches et Pratiques sur les ACTivités

Référence électronique

Amélie Sandoval et Katia Kostulski, " "Si on ne me fournit pas mes engins, après je peux plus rien faire moi" : les apports de l'analyse de l'activité à la compréhension des processus d'engagement et de désengagement au travail », Activités [En ligne], 18-2 | 2021, mis en ligne le 15 octobre 2021, consulté le 07 avril 2022. URL : http://journals.openedition.org/activites/6835 ; DOI : https://doi.org/ 10.4000/activites.6835

Ce document a été généré automatiquement le 7 avril 2022.

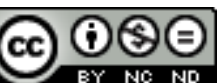

Activités est mis à disposition selon les termes de la licence Creative Commons Attribution - Pas d'Utilisation Commerciale - Pas de Modification 4.0 International. 


\section{"Si on ne me fournit pas mes engins, après je peux plus rien faire moi" : les apports de l'analyse de l'activité à la compréhension des processus d'engagement et de désengagement au travail}

"If you don't give me the required equipment, I won't be able to do any thing": the contribution of the analysis of activity to the understanding of commitment and disengagement processes at work"

Amélie Sandoval et Katia Kostulski

NOTE DE L'ÉDITEUR

Article soumis le 02/06/2021, accepté le 04/09/2021

\section{Introduction}

1 L'engagement au travail fait l'objet de nombreuses publications, notamment en psychologie du travail et des organisations, en psychologie positive de la santé ou du travail, ou encore en sciences de gestion. Ces dernières conceptualisent le plus souvent l'engagement ${ }^{1}$ comme un comportement vis-à-vis d'une ou plusieurs dimensions du travail ou de l'emploi, ou comme des états affectifs et émotionnels qui caractérisent de façon durable le sujet au travail. Elles ne pensent que rarement les liens entre l'engagement - ou le désengagement - et l'activité des professionnels. Les 
manifestations d'engagement et de désengagement des professionnels, que nous avons pu rencontrer et voir se développer au cours d'une intervention en clinique de l'activité dans un service public de la propreté, nous ont amenées à interroger ces différentes approches pour nous tourner vers d'autres perspectives théoriques permettant de penser l'engagement et le désengagement dans leurs rapports avec l'activité réelle des professionnels.

Dans une première partie théorique, nous exposerons les deux principales approches de l'engagement rencontrées dans les travaux scientifiques menés sur le sujet, puis la façon dont la tradition francophone d'analyse du travail, la psychopathologie du travail et les cliniques du travail permettent d'aborder l'engagement et le désengagement au travail dans leurs liens avec l'activité et à travers leurs significations dans des situations concrètes de travail. Nous mobiliserons ensuite une intervention menée dans un service municipal de la propreté d'une grande ville, et des analyses tirées de cette intervention, afin d'explorer plus précisément comment engagement, désengagement et activité peuvent s'articuler. Cette intervention, qui avait pour origine une préoccupation de la direction du service sur l'absentéisme des éboueurs, nous a en effet amenées à co-analyser avec un collectif de chefs d'équipe leur activité et à approcher alors la façon dont ils s'engageaient dans la réalisation de leurs tâches, ou au contraire s'en désengageaient, en lien avec le contexte immédiat - les possibles et les impossibilités concrètes dans la situation -, avec la ligne hiérarchique, ou encore avec l'organisation du travail, laquelle, nous le verrons, se révèle parfois peu propice à l'efficacité de leur action. Nous conclurons sur ce que l'analyse de l'activité permet d'apporter à la compréhension des dynamiques d'engagement et de désengagement des professionnels.

\section{Différentes approches théoriques de l'engagement au travail}

\subsection{L'engagement comme lien à une (ou des) cible(s) spécifique(s)}

3 Les premiers travaux sur l'engagement en milieu professionnel se développent aux États-Unis, en sociologie puis en psychologie, dans les années 1950-1960. En psychologie du travail, cette question est d'abord abordée à travers le concept d'engagement organisationnel (Organizational Commitment). Défini par Mowday, Porter et Steers (1982) comme un engagement affectif, il renvoie à la force relative de l'identification et de l'implication de quelqu'un dans une organisation particulière. Il se caractérise par son acceptation et sa croyance dans les buts et les valeurs de cette organisation, sa volonté d'exercer des efforts à son profit et son désir d'en rester membre. Meyer et Allen (1991) proposent par la suite un modèle tridimensionnel de l'engagement organisationnel en distinguant une composante affective, une composante calculée et une composante normative. L'engagement organisationnel renvoie ainsi à l'attachement de l'individu vis-à-vis de l'organisation (engagement affectif), à sa représentation des coûts et avantages associés au fait de quitter ou de rester dans l'organisation (engagement calculé ou de continuité), et à son sentiment d'obligation morale de devoir agir selon les buts et valeurs de l'organisation (engagement normatif). Malgré des critiques et quelques remaniements (p. ex. Klein et al., 2012 ; Paillé, 2002, 2005 ; Solinger, van Olffen, \& Roe, 2008), ces trois dimensions restent fréquemment mobilisées dans les 
travaux actuels (p. ex. Desrumaux, Léoni, Bernaud, \& Defrancq, 2012; Paillé 2012; Vandenberghe, 2016). On notera que, progressivement, dans ces différents travaux, l'engagement ne se déploie plus forcément en direction de l'organisation, mais peut aussi concerner une autre entité ou « cible » du milieu professionnel (Klein, Molloy, \& Brinsfield, 2012; Meyer, \& Herscovitch, 2001), comme par exemple le supérieur hiérarchique, le groupe de travail, la profession exercée ou encore les clients (Paillé, 2009).

4 Dans ces recherches, l'engagement organisationnel est actif dans le sens où il relève à la fois de croyances, d'opinions ou d'états psychologiques ressentis vis-à-vis d'une «cible» particulière, et de comportements ou d'actions induits par ces croyances, opinions ou états. Il est aussi au moins pour une partie "choisi » (et pas seulement induit par les caractéristiques ou particularités de la cible) par des individus qui effectuent des choix conscients et volontaires. Si certaines caractéristiques du travail ou du poste comme la variété des tâches ou l'autonomie dans l'exercice de l'emploi (Louche, 2017) peuvent être identifiées parmi les multiples antécédents à l'engagement, l'activité en tant que telle, comprise comme ce qui est fait par le sujet pour effectuer la tâche (Falzon, 2004 ; Leplat, \& Hoc, 1983), y compris - nous y reviendrons - dans ses dimensions non réalisées (Clot 2004), n'apparaît pas dans ces modélisations. L'engagement organisationnel aurait par contre des effets sur elle en favorisant notamment l'effort au travail et la performance (Louche, op. cit.).

\subsection{L'engagement comme état psychologique de la personne}

5 De façon plus récente, se développent des travaux sur l'engagement au travail dans la perspective de la psychologie positive. L'engagement au travail (Work Engagement) y est défini comme un état affectif et motivationnel de bien-être au travail, positif et épanouissant, qui se caractérise par la vigueur, le dévouement et l'absorption, un haut niveau d'énergie et une forte identification au travail. Les professionnels engagés ont de l'énergie, de l'enthousiasme et se sentent complètement «pris » dans leur travail (e.g. Bakker, \& Leiter, 2010 ; Schaufeli, 2013). L'engagement au travail est conceptualisé comme relevant du bien-être au travail et s'opposant au burn-out (Maslach, \& Leiter, 1997/2011). Le burn-out peut alors être vu comme une érosion de l'engagement au travail qui se caractérise par l'épuisement émotionnel, psychique et physique, le cynisme (ou la dépersonnalisation) et un sentiment d'inefficacité professionnelle (Bakker, Schaufeli, Leiter, \& Taris, 2008).

6 L'engagement n'est pas ici un lien ou une force qui relie un individu et une ou plusieurs dimensions de travail, mais un état affectif et cognitif persistant et complet. Ainsi il n'est pas axé sur un objet, un événement, un individu ou un comportement particulier et caractérise plus globalement l'«état psychologique » d'une personne (Schaufeli, Bakker, \& Salanova, 2006). Schaufeli et Bakker (2010) distinguent l'engagement au travail de l'engagement organisationnel : ce dernier est pour eux un lien d'attachement et d'identification entre un individu et une organisation, tandis que l'engagement au travail découlerait plutôt du rôle professionnel et des caractéristiques du travail. Il est notamment favorisé par la quantité et la diversité des ressources disponibles pour le professionnel.

7 Ces ressources relèvent pour partie des dimensions physiques, sociales et organisationnelles de l'emploi qui peuvent contribuer à réduire les exigences du travail 
et les coûts associés, permettre à la personne d'atteindre ses objectifs professionnels, et stimuler l'apprentissage et le développement personnel. Les recherches empiriques mettent notamment en avant le rôle du soutien social des collègues et des encadrants, du «feedback» sur la performance, du climat organisationnel, de la variété des compétences sollicitées, des occasions d'apprendre ou encore de l'autonomie. Ces ressources relèvent aussi de la personne ou "de son capital psychologique ", et notamment de son sentiment d'efficacité personnelle, son estime de soi, son optimisme ou sa résilience (Bakker et al., 2008). Là encore, l'activité en tant que ce qui est fait dans des situations concrètes de travail n'apparaît pas : ce qui joue sur l'engagement selon ces auteurs renvoie plutôt à l'emploi dans ses dimensions prescrites et générales, ou à ce que serait sur un plan psychologique l'individu indépendamment de l'activité dans laquelle il est engagé.

Si les deux perspectives présentées divergent dans leur façon de concevoir l'engagement au travail, il est notable que pour l'une comme pour l'autre l'engagement ne se construit pas dans l'activité, mais a, le plus souvent, des retombées positives sur elle : il est bon pour l'individu et l'organisation, il favorise aussi bien la santé et le bienêtre au travail que la productivité et la performance de l'organisation. Par ailleurs, elles conçoivent l'une et l'autre l'engagement comme une dimension stable et mesurable et développent des échelles permettant de le mesurer. Nos propres observations et analyses viennent interroger ce caractère stable et mesurable, avec des professionnels pour lesquels - nous y reviendrons - il est souvent difficile de dire s'ils sont engagés "en général» ou "en moyenne», et ce justement parce que l'engagement et le désengagement varient en fonction de leur activité. Comme le soulignait déjà Kahn (1990) dans ses travaux sur les conditions psychologiques de l'engagement et du désengagement au travail, un professionnel peut être engagé à certains moments et ne l'être pas à d'autres, être engagé dans la réalisation d'une tâche et ne l'être pas dans celle d'une autre.

\subsection{Vers un engagement dans et par l'activité ?}

Il paraît alors intéressant de regarder plus précisément dans quelles situations les travailleurs, individuellement et collectivement, s'engagent dans leur travail, et plus précisément ce qui, dans leur activité, dans les contraintes et les ressources qu'ils y rencontrent, peut développer de l'engagement et/ou du désengagement.

10 Nous verrons à partir d'un exemple tiré d'une intervention sur le terrain comment un professionnel peut paraître, à première vue, se désengager de la réalisation d'une tâche et se révéler au contraire, au fil de la co-analyse de son activité, vivre une configuration plus complexe : une configuration où se mêlent engagement et désengagement, en lien notamment avec les contradictions qui traversent ce professionnel, entre ce qu'on lui demande de faire, ce qu'il voudrait faire, et ce qu'il parvient à faire en mobilisant des moyens en grande partie non prévus par l'organisation du travail. Pour entrer dans cette conflictualité qui le tiraille entre engagement et désengagement, et tenter de la comprendre, il convient de regarder précisément ce qui se joue dans l'activité et comment les professionnels se mobilisent de façon variable et parfois ambivalente pour faire ce qui est à faire, selon l'organisation, ou parfois... selon leur point de vue propre.

11 L'activité est ici à entendre, comme «ce qui est fait, ce qui est mis en jeu par le sujet pour effectuer la tâche» (Falzon, 2004, p. 24). Elle émerge de l'interaction entre un 
sujet et une tâche (Leplat, \& Hoc, 1983) et ne dépend pas seulement des buts et des conditions de réalisation prescrits par l'organisation, mais aussi des buts et moyens que le sujet se fixe ou construit. Dans ce modèle, le professionnel est « un sujet actif, engagé à la fois dans la réalisation du travail et dans la préservation/transformation de luimême. [Ce modèle] s'oppose à celui d'un sujet exécutant, passif, "effecteur" d'une tâche prescrite. » (Falzon, 2013, p. 5). Ici, une forme d'engagement paraît être constitutive de la réalisation de l'activité. Les cliniques du travail qui s'appuient sur l'ergonomie de langue française et la psychopathologie du travail s'intéressent très précisément à ce qui va être fait réellement et mobilisé objectivement et subjectivement par les professionnels dans leur travail, avec l'idée qu'il existe un irréductible écart entre le travail prescrit et l'activité réelle (Lhuilier, 2006 ; Molinier, 2006). Le travail est toujours une confrontation à ce qui n'est pas donné, prévu ou attendu dans la situation, et un " engagement face à ce qui n'est pas maîtrisé » (Davezies, 1994, p. 5). De ce point de vue, l'engagement relève de cette mobilisation, en pensées et en actes, du professionnel qui, face à ce qui fait problème ou résiste, cherche à agir.

La clinique de l'activité reprend cette distinction entre la tâche prescrite et l'activité, en y ajoutant une distinction entre l'activité réalisée et le réel de l'activité : à côté de ce que réalise le professionnel, on prend en compte dans l'analyse de l'activité des dimensions non réalisées de celle-ci, comme ce qu'il aurait voulu faire, ce qu'il cherche à faire sans y parvenir, ce qu'il renonce à faire, ce qu'il fait sans le vouloir, etc. (Clot, 2004). Cette perspective modélise l'activité comme triplement dirigée vers trois pôles que sont le sujet, l'objet de la tâche et les autres (Clot, 2004, 2008). Elle est ainsi polycentrique et traversée par des contradictions, des tensions et des conflits, à l'intérieur de chaque pôle et entre ces trois pôles. Agir, c'est alors s'appuyer plus ou moins sur un pôle ou l'autre, arbitrer entre diverses possibilités et priorités, combiner différentes exigences et contraintes à l'intérieur d'une même activité ou entre différentes activités, dans des formes variables de mobilisation de soi. On peut alors imaginer que l'engagement et le désengagement peuvent se déployer dans une activité et pas dans une autre, ou caractériser une des dimensions de l'activité et pas l'activité dans son ensemble. Un professionnel peut par exemple refuser de faire certaines choses pour en faire d'autres ; à l'intérieur d'une même activité, il peut s'engager en direction d'un destinataire et pas d'un autre; etc. Cette conception de l'activité permet de se représenter comment un professionnel n'est pas « engagé » ou " désengagé », de façon générale et durable, mais peut être l'un puis l'autre, ou même l'un et l'autre, dans une configuration globale singulière et complexe, composée d'une superposition de mouvements ou actes d'engagement et de désengagement.

\subsection{L'engagement et le désengagement dans l'activité : des conduites chargées de significations?}

13 Si on situe l'engagement et le désengagement comme se déployant dans l'activité, on peut s'interroger sur leurs significations, voire sur leur fonction, dans cette activité, et sur la variabilité de celles-ci. Par exemple, si aller au travail est un signe d'engagement, quelquefois ne pas y aller est aussi un signe d'engagement : se désengager à un moment peut être une nécessité passagère pour être plus présent et plus disponible à un autre moment. 

permettent de mieux comprendre la fonction comme la signification de l'engagement et du désengagement. Dans la lignée des travaux de Veil sur l'absentéisme (1960/2012), l'engagement et le désengagement peuvent s'appréhender comme des conduites dont la signification est loin d'être univoque. Elle est à chercher dans la rencontre entre un individu et une situation, et pas seulement dans l'un ou l'autre, au croisement d'éléments individuels, sociaux et organisationnels. Ils peuvent alors se comprendre comme des formes de régulation de l'activité et, pour le dire à la manière de Claude Veil, les désengagés comme les absents «n'ont pas toujours tort » (Veil, op. cit., p. 167). A l'instar de la présence et l'absence au travail telles qu'elles sont analysées par Veil, l'engagement et le désengagement ne sont ni «bons", ni " mauvais » en soi, comme l'avance fréquemment la littérature, mais plus ou moins adaptés, en fonction de ce qu'ils peuvent permettre ou non dans la situation. Ils traduisent alors une adaptation ou une inadaptation « subjective et relative» (Veil, op. cit., p. 166).

Dans certaines configurations d'engagement et de désengagement, ce sont davantage des mécanismes défensifs qui sont à l'œuvre. La psychodynamique du travail a notamment montré comme le désengagement ou le retrait pouvaient être des façons de tenir face aux difficultés rencontrées et à la souffrance psychique générée par les contraintes du travail. Duarte (2017) identifie par exemple des formes individuelles et collectives de «retrait (ou repli) défensif» (pp.177-179, pp.379-380, p. 416), par lesquelles les salariés tentent de se protéger du risque de surcharge, des effets de l'expérience douloureuse du déni de reconnaissance et de l'appauvrissement du travail, et plus largement de la souffrance éthique générée par le fait de ne pas parvenir à maintenir un travail de qualité. L'ethos professionnel est abandonné, le retrait est alors une réponse au risque que constitue l'engagement. Viviers (2014), quant à lui, identifie des formes intermédiaires dans lesquelles les professionnels ne se retirent pas complètement de leur travail, mais tentent de modérer leurs investissements. Pour autant, le surinvestissement aussi peut s'ériger en défense, comme dans les cas des conduites d'auto-accélération ou d'hyper activité où l'engagement intense permet de ne pas penser à ce qui fait souffrir (Dejours, 2004 ; Molinier, 2006).

et le désengagement peuvent encore servir des pratiques de résistances quotidiennes (Efros, \& Schwartz, 2009 ; Lhuilier, \& Roche, 2009 ; Roche, 2016). Désobéir ou au contraire appliquer «à la lettre " certaines consignes, ignorer les demandes implicites, ne plus chercher à s'ajuster aux inattendus, et finalement ne plus se mobiliser pour combler l'écart existant entre le prescrit et le réel sont aussi des façons d'enrayer le fonctionnement de l'organisation et de tenter de peser, même à petite échelle, sur ce qui s'y passe. La grève du zèle, par exemple, qui consiste pour les professionnels à s'engager dans une application stricte de la règle en retirant « tout ce qu'[ils] ajoutent à l'organisation prescrite du travail pour être efficace; tout ce qu'ils mettent en œuvre individuellement et collectivement et qui ne relève pas de l'“exécution" " (Dejours, 1998, p. 31), provoque souvent par là même des dysfonctionnements, des blocages, une "paralysie de la production ou des erreurs majeures dans le déroulement du procès de travail » (Duarte, 2017, p. 40). Cette forme d'engagement/désengagement constitue alors parfois un moyen de résister, plus ou moins efficacement, à des prescriptions et décisions organisationnelles.

Les conduites d'engagement et de désengagement peuvent ainsi revêtir différentes significations, pas toujours celles qu'on pourrait leur donner a priori, et des 
significations qui peuvent entrer en contradiction les unes avec les autres. Le désengagement de certaines tâches - quand il constitue une façon d'essayer de faire réagir l'organisation - peut par exemple aussi traduire l'engagement de professionnels qui n'ont pas renoncé à donner du sens à leur travail et à améliorer le fonctionnement de la structure qui les emploie. Tenter de comprendre l'engagement et le désengagement suppose à la fois d'examiner précisément avec les professionnels l'activité menée, ses conditions, ses contraintes et ses ressources, mais aussi ce que s'engager et se désengager peuvent vouloir dire de ce qu'ils vivent et cherchent à faire au cours de cette activité.

Pour étayer nos propos, nous nous appuierons dans la partie qui suit sur l'intervention évoquée plus haut et rentrerons dans le détail d'une dynamique complexe et singulière d'engagement et de désengagement, à laquelle l'analyse de dialogues extraits de deux cadres de co-analyse de l'activité permet, pour partie, d'accéder.

\section{Une approche des dynamiques d'engagement/ désengagement au travail par l'analyse de l'activité : l'exemple d'une intervention dans un service public de la propreté}

19 Après avoir brièvement présenté l'intervention sur laquelle nous nous appuierons, nous montrerons comment celle-ci a permis d'identifier des dysfonctionnements dans le service et des tensions dans le métier chefs d'équipe, ainsi qu'un ensemble de manifestations d'engagement et de désengagement lié à ces dysfonctionnements et tensions. Nous nous arrêterons alors sur un exemple permettant de saisir comment l'analyse d'une activité précise donne des éléments de compréhension d'une configuration pour le moins singulière d'engagement/désengagement d'un professionnel.

\subsection{Méthodologie de l'intervention}

\subsubsection{Une commande sur l'absentéisme et l'activité des chefs d'équipes}

L'intervention dont il sera question ici a été menée en référence au cadre théorique et méthodologique de la clinique de l'activité (Clot, 2004 ; 2008 ; Kostulski, 2010, 2011). Celle-ci se centre sur les rapports entre subjectivité et activité et vise au développement $\mathrm{du}$ pouvoir d'agir des professionnels. Dans cette perspective développementale, il s'agit d'intervenir, à partir de demandes émanant de milieux professionnels, en construisant des cadres d'analyse et d'élaboration collectives qui permettent aux professionnels de dire quelque chose de ce qu'ils font, mais aussi de ce qu'ils ne font pas, ou encore de ce qu'ils pourraient ou voudraient faire autrement. C'est ainsi toute "l'épaisseur " de leur activité réelle que l'on cherche à mettre en discussion, avec pour objectif d'élargir les ressources et les possibilités des professionnels face aux situations qu'ils rencontrent, de leur permettre d'expérimenter de nouvelles façons de faire de manière à «élargir leur rayon d'action, leur pouvoir d'agir sur leur milieu et sur eux-mêmes " (Clot, 2008, p. 182). Une telle intervention cherche à permettre aux professionnels et aux organisations de travail de développer le 
sens et l'efficience de leur action et d'initier des transformations allant dans le sens de la santé et de l'efficacité au travail.

Pour ce qui concerne notre intervention, c'est une commande émanant de la direction d'un service municipal de la propreté qui nous a amenées à travailler pendant trois ans (2015-2018) avec des chefs d'équipe du nettoiement ${ }^{2}$ et leur hiérarchie. Ce service a en charge la propreté des rues et la collecte des ordures et la demande de départ portait sur l'absentéisme des éboueurs dans le service. De l'ordre de $13 \%$ depuis plusieurs années, le taux d'absentéisme peut avoisiner les $20 \%$ dans certains ateliers de la propreté $e^{3}$ et pose d'importants problèmes d'organisation du travail qui ont des effets à la fois sur la qualité du travail réalisé et sur la santé des éboueurs : entre autres, les tâches ne peuvent pas être toutes effectuées, la charge de travail se reporte sur les présents et il devient difficile de faire tourner les tâches les plus pénibles. Un ensemble d'hypothèses et de pistes d'actions possibles sur l'absentéisme circule au sein de la direction, et parmi elles celle du rôle que pourrait jouer l'encadrement de proximité dans la prévention de l'absentéisme. C'est cette piste que veut explorer la direction quand elle fait appel à nous: elle souhaite que cette question de l'absentéisme soit travaillée non pas directement avec les éboueurs, mais avec leurs chefs d'équipe. Le chef d'équipe est vu ici comme le principal acteur de la mise au travail des agents, et de leur présence même au travail chaque matin.

Il est à noter qu'une première intervention de notre équipe de recherche a eu lieu dans ce même service entre 2011 et 2014 (voir Bonnemain, 2015). Elle a notamment permis d'identifier que les chefs d'équipe avaient bien comme préoccupation de «faire revenir les éboueurs le lendemain $»^{4 \& 5}$, qu'ils développaient des manières de s'y prendre, notamment dans la répartition des tâches, pour favoriser cela, mais qu'ils rencontraient aussi un ensemble de problèmes dans leur propre activité qui mettaient à mal leurs efforts en ce sens. Lors du travail de notre commande de départ et dans la continuité de l'intervention précédente, nous arrivons à la proposition (1) de travailler avec les chefs d'équipe sur des difficultés concrètes qu'ils rencontrent dans leur propre activité et qui ont des conséquences sur le travail des éboueurs, (2) de chercher collectivement des solutions à ces difficultés dans des cadres de travail qui permettront aux chefs d'équipe de travailler à la fois entre pairs et avec leur propre hiérarchie. La perspective est d'expérimenter ainsi de nouvelles modalités de travail collectif qui permettraient de construire et d'expérimenter des solutions aux problèmes rencontrés par les chefs d'équipe. Il s'agit finalement de chercher à développer leur possibilité d'agir sur l'organisation du travail pour construire un cadre de travail qui favorise l'engagement des éboueurs.

\subsubsection{Le dispositif d'intervention}

23 Pour ce faire, nous avons travaillé dans deux divisions de la propreté, avec deux collectifs de chefs d'équipe, sur deux activités particulières qui leur posaient problème et fragilisaient leur fonction d'encadrement. Le choix de ces activités a été fait par les chefs d'équipe en concertation avec leur hiérarchie : l'objectif était qu'il y ait un accord de départ sur ce qu'il était pertinent de travailler dans chacune des divisions. Dans une division, a été choisie l'activité de signalement et transmission (d'informations, d'anomalies...) depuis le terrain, dans l'autre celle du traitement des plaintes des usagers. Ces deux activités ont fait l'objet de co-analyses qui ont amené les 
professionnels à dire quelque chose de ce qu'ils faisaient, à le discuter et à le débattre entre pairs.

Ces co-analyses ont été reprises dans des discussions internes aux divisions dans le cadre de groupes de travail transverses, au sein desquels les chefs d'équipe ont pu présenter et discuter leurs problèmes, leurs analyses ainsi que les pistes de solution envisagées, avec leur hiérarchie et avec d'autres fonctions de la division qui pouvaient être concernées par les questions soulevées. Ce travail transverse a pu donner lieu à un élargissement de points de vue et à l'élaboration de solutions concertées pour certains de ces problèmes, solutions qui ont pour une part été expérimentées et validées.

Enfin un troisième niveau de travail a complété le dispositif : nous avons mis en place un comité de pilotage, constitué dès le début de l'intervention, réunissant la commanditaire de l'intervention et des membres de son équipe, des responsables de la prévention des risques professionnels dans le service, et des représentants à la fois des collectifs des chefs d'équipe et des divisions. Il s'agissait dans ce comité de faire circuler, cette fois-ci à l'échelle du service, les analyses réalisées et les questions soulevées avec les chefs d'équipes, ainsi que la façon dont elles étaient travaillées dans les différentes divisions et dans la ligne hiérarchique. Différents points de vue s'y sont donc rencontrés, parfois confrontés, pour chercher à construire collectivement à la fois l'orientation de l'intervention en cours et de possibles solutions face aux difficultés collectivement travaillées. Dans le dialogue entre les différents participants, les membres du comité de pilotage ont pris un certain nombre de décisions concertées concernant notamment des actions à expérimenter dans les divisions.

Dans ces différents cadres de travail du dispositif, il s'agissait de pouvoir mettre en dialogue une pluralité de points de vue, de confronter ce qui faisait désaccord, et de chercher à construire des compromis et des pistes d'action acceptables. La conflictualité est ainsi centrale dans le travail développemental réalisé en intervention, aussi bien dans les collectifs de pairs que dans le comité de pilotage. Il faut entendre ici le conflit «non pas comme un but, mais comme un moyen d'intelligence et de création collectives » (Clot, \& Quillerou-Grivot, 2014, p. 142).

Le travail de co-analyse de l'activité effectué dans cette intervention s'est fait à partir d'observations, de réunions collectives, d'autoconfrontations simples et d'autoconfrontations croisées (désormais ACS et ACC). Il convient ici de rappeler les principes de cette méthode d'analyse de l'activité. La méthode des analyses en autoconfrontations croisées (Bonnemain, \& Clot, 2017; Clot, Faïta, Fernandez, \& Scheller, 2000) consiste à confronter des professionnels à des traces de leur activité. Dans cette intervention précise, ces traces étaient à la fois des traces filmées et des documents de travail ${ }^{6}$. Les professionnels s'y confrontent une première fois individuellement, seul avec l'intervenant. C'est l'occasion pour eux d'une première analyse au cours de laquelle ils peuvent prendre pour objet ce qu'ils font, s'en étonner, tenter de l'expliquer, dire quelque chose des contraintes et des ressources de la situation, ou encore de ce qui se joue dans leur façon de faire. Lors de l'ACC, s'ouvre un second cadre d'analyse dans lequel deux professionnels se confrontent à leurs traces d'activité respectives et peuvent alors discuter leurs convergences et divergences de façons de faire et de points de vue. Les analyses réalisées en ACS et en ACC sont ensuite reprises dans des séances collectives rassemblant tous les chefs d'équipe qui peuvent alors poursuivre l'analyse collective et notamment sélectionner les points de difficulté ou de divergence qui leur paraissent importants à travailler ensemble, puis avec leurs 
propres encadrants et dans le comité de pilotage. Par cette méthode, sont à l'œuvre " différents cadres de dialogue [qui] permettent ainsi le développement de l'analyse : seul, avec un collègue lui-même confronté à ses propres images, avec le collectif associé à l'intervention dans son ensemble. » (Kostulski, 2011, p. 82-83).

\subsection{Premières analyses : dysfonctionnements dans le service et tensions dans le métier}

Ce travail a permis d'identifier des dysfonctionnements à l'intérieur des divisions et un ensemble de tensions dans le métier des chefs d'équipe qui doivent régulièrement arbitrer entre différentes options possibles, pas toujours compatibles entre elles. Par exemple, les co-analyses autour du traitement des plaintes ont révélé que, du point de vue des chefs d'équipe, les plaintes n'étaient pas toujours «justifiées » et n'étaient pas non plus toujours traitées sur le terrain. Certaines d'entre elles se sont révélées être sans solution, ou nécessiter des actions que les chefs d'équipe ne parvenaient pas à mettre en place (faute de moyens matériels et humains, ou faute d'identifier le bon interlocuteur pour faire aboutir sa demande d'action). Le manque de réactivité du service ainsi qu'une mauvaise circulation de l'information ont aussi été identifiés comme points de blocage dans le traitement des plaintes. Enfin, ont été aussi distingués, d'une part, un traitement administratif de la plainte qui consiste à répondre au plaignant en lui donnant des informations sur la façon dont a été prise en charge la zone qui fait l'objet de la plainte (en indiquant par exemple combien de fois elle a été lavée ou balayée au cours des derniers jours) et, d'autre part, un traitement technique de la plainte qui consiste à agir sur le terrain en mettant en place des actions de nettoyage, correctives et/ou préventives, sur une zone particulière.

Du côté du métier, les chefs ont mis au jour notamment deux tensions particulières. La première concerne leur objectif partagé de réactivité. Être présent sur le terrain et réagir rapidement est important dans le métier, mais se révèle avoir des effets pervers en développant les attentes et demandes des usagers, ainsi que les incivilités. D'une certaine façon, être efficace dans le traitement des plaintes peut encourager les usagers à formuler des plaintes puisque celles-ci sont suivies d'effets. De même, nettoyer rapidement les dépôts sauvages faits par les riverains incite ceux-ci à continuer à en faire puisque quel que soit leur mode de dépôt des ordures (dans des bacs prévus à cet effet ou directement sur le trottoir), elles sont de toute façon ramassées dans des délais rapides. La seconde tension concerne le dilemme entre propreté optimale et satisfaction des riverains. Le fait de répondre aux plaintes, et finalement d'allouer plus de moyens à certains endroits, amène les chefs d'équipe à développer ce qu'ils appellent un "service à la carte». Celui-ci peut permettre de satisfaire les plaignants, mais empêche de fait d'assurer un bon niveau de propreté globale sur leur secteur, avec un traitement à peu près égal entre les lieux. En dehors de certains axes qui (parce que très passants ou commerçants, ou symboliques comme devant les mairies) peuvent jouir d'un traitement renforcé, il conviendrait, selon les chefs d'équipe, d'assurer un traitement qui soit le même partout. Quand la satisfaction des usagers implique ce qu'ils estiment être des traitements de faveur, alors elle n'est plus compatible avec leur objectif de propreté globale optimale. 


\subsection{Différents mouvements d'engagement et de désengagement} désengagement : engagement et désengagement dans l'intervention elle-même, avec des professionnels pas toujours faciles à mobiliser dans les différents cadres de l'intervention et une mobilisation parfois instable, mais aussi engagement et désengagement dans leur activité, d'après ce que les professionnels nous en ont donné à voir ou dit dans les temps d'observation et d'analyse. Précisons-le d'emblée, cet engagement et ce désengagement ne sont pas à prendre comme des dispositions individuelles et encore moins comme des inclinaisons morales qui relèveraient des personnes. Ils sont apparus dans des situations particulières, dans lesquelles ils se développaient, voire s'imposaient aux différents protagonistes, comme des façons possibles ou inévitables de faire, de répondre, de se positionner, face à une demande ou une prescription. En cela, ces mouvements renvoient à la fois à des obstacles et des ressources que les professionnels rencontrent dans les situations de travail et les activités au sein desquelles ils se déploient, et à la façon dont ces professionnels vivent ces situations et tentent d'y agir.

31 Ce que les chefs d'équipes disent, dans les co-analyses, de ce qu'ils font, et les tensions qu'ils mettent au jour, permettent d'identifier plus précisément ce qui peut générer de l'engagement, mais aussi ce qui peut générer du désengagement ou encore limiter l'engagement initial des professionnels. Dans l'analyse de l'activité de traitement des plaintes, par exemple, nous avons pu voir que certaines tâches étaient investies (comme celles en lien avec le traitement technique des plaintes sur le terrain) et que d'autres ne l'étaient pas ou peu (comme celles en lien avec le traitement administratif des plaintes attendu par la hiérarchie). Les discussions ont aussi montré que certaines plaintes étaient traitées, d'autres non, en fonction de critères d'évaluations qui se sont précisés dans les dialogues. Ainsi certaines plaintes sont mises de côté car considérées comme injustifiées, comme certaines plaintes dites justifiées peuvent aussi l'être quand les professionnels pensent ne pas avoir les moyens de les traiter. Les arbitrages généraux peuvent varier en fonction de la nature de la plainte (avec des discours génériques du type : «en général les plaintes je ne les traite pas, mais celle-ci oui, car elle me paraît particulièrement justifiée »). Et pour une même tâche, l'engagement peut aussi être changeant en fonction de la personne à qui s'adresse le professionnel (avec des discours génériques du type: «je réponds à certaines demandes administratives concernant la plainte quand elles proviennent de mon supérieur direct, mais pas quand elles proviennent de la direction de ma division»). Loin de faire toujours pareil et d'appliquer les procédures à la lettre, les chefs d'équipe régulent leurs interventions et mobilisations pour faire ce qu'on leur demande de faire, notamment en fonction de l'objet de l'activité, de ses destinataires et des moyens disponibles pour le faire.

Les différentes tensions dans le métier des chefs d'équipe comme celles décrites précédemment traduisent des arbitrages constants entre faire et ne pas faire, avec des dosages complexes et changeants de leur investissement dans la réalisation de la tâche. Ainsi, si les chefs d'équipe sont parfois très réactifs pour répondre à des plaintes, ils se retiennent à d'autres moments d'intervenir pour éviter les effets pervers ou favoriser une répartition égalitaire des moyens humains et matériels sur le secteur géographique dont ils assurent la propreté. Les échanges dans les collectifs montrent que les arbitrages faits paraissent fortement liés à l'expérience qu'ont les chefs d'équipe du 
fonctionnement de leur organisation et aux représentations qu'ils se font de ce fonctionnement (et qu'ils se transmettent entre eux). Ainsi certaines conduites de désengagement peuvent se comprendre comme le résultat de l'expérience et/ou du sentiment partagé que certaines façons de se mobiliser pour réaliser la tâche sont inutiles et même parfois contre-productives.

Enfin, ce que nous avons aussi pu voir dans les observations et les analyses menées, c'est que les chefs d'équipe pouvaient s'engager au quotidien pour faire leur travail au mieux, malgré des conditions d'exercice difficiles, malgré le manque de moyens (humains, techniques), et dans certains cas malgré l'absence de soutien ou de reconnaissance (des supérieurs, des usagers, voire des pairs), en prenant sur soi, en dérogeant à la règle, en inventant de nouveaux moyens "clandestins", etc. Le désengagement par rapport à certaines demandes nous a même paru parfois pouvoir être compris comme un moyen pour s'engager par rapport à d'autres demandes, ou pour maintenir leur engagement à plus long terme. Par exemple quand des tâches administratives sont désinvesties, cela peut être pour avoir plus de temps pour se mobiliser dans d'autres tâches (comme le travail sur le terrain). Se désengager à court terme ou à un endroit de l'activité peut aussi être une façon de tenter de créer un cadre de travail dans lequel s'engager à plus long terme ou sur un plan plus général. C'est le cas quand le désengagement traduit une forme de résistance aux rapports de pouvoir comme dans certaines conduites observées ou décrites par nos interlocuteurs : ne pas faire, ou faire a minima, est alors parfois une tentative délibérée de faire gripper la machine organisationnelle et de ne pas en subir le fonctionnement. Le désengagement de l'activité traduit alors peut-être une forme d'engagement qui vise à l'instauration d'autres rapports et d'autres fonctionnements dans l'organisation de l'ordre d'une « résistance souterraine » (Scott, 1990/2006).

Comme nous allons le voir en nous arrêtant plus particulièrement sur un exemple, de nombreuses nuances apparaissent et différents éléments relevant des niveaux individuel, collectif et organisationnel interviennent, quand on cherche à décrire et à comprendre la façon dont les personnes s'engagent ou se désengagent dans la réalisation de leur activité.

\subsection{Un exemple de configuration particulière d'engagements/ désengagements}

\subsubsection{La plainte de l'avenue du Layon}

Nous allons nous arrêter maintenant sur une plainte, et plus précisément sur l'analyse qu'un chef d'équipe et un de ses collègues font du traitement de cette plainte particulière dans le cadre de différents dialogues. Les analyses de ces dialogues s'inscrivent dans la perspective d'une psychologie concrète du langage (Kostulski, 2011) et s'appuient sur une méthodologie d'analyse langagière de l'énonciation que nous avons construite, en nous appuyant principalement sur les travaux en linguistique de Vion et Rabatel (Rabatel, 2008 ; Vion, 2004, 2011). Ces analyses prennent pour objet à la fois ce que les professionnels disent de ce qu'ils font, et leur rapport subjectif à ce qu'ils disent en termes de positionnement et d'implication dans l'énonciation. Elles permettent de faire des inférences et des hypothèses sur l'engagement et le désengagement des professionnels et la façon dont ils se développent dans l'activité. Nous ne pourrons pas décrire plus ici la construction de cette méthodologie (cf. 
Sandoval, 2020, pour le détail de la méthodologie des analyses énonciatives dans la recherche), mais un tableau en annexe reprend les principaux phénomènes énonciatifs et indices sur lesquels nous nous sommes appuyées. À l'intérieur des extraits qui sont utilisés dans la suite de l'article, les principaux indices mobilisés sont indiqués de la façon suivante :

- surligné gris : les pronoms, les modalisateurs principaux et les commentaires réflexifs

- souligné : les autres traces de subjectivité présentes dans les énoncés et mobilisées dans l'analyse

- en gras : les éléments de contenu portant sur l'objet de l'activité analysée, et sur l'activité réalisée et/ou réellement menée (actions entreprises, mobiles, buts...)

La plainte de l'avenue du Layon est une plainte récurrente, régulièrement émise par la gérante d'une boutique de vêtements (au moins 3 fois sur les 6 derniers mois au moment de l'ACS). Cette dernière trouve que les abords de son magasin sont «sales» (présence de détritus, mégots, etc.). Cette plainte est une de celles retenues avec Pascal pour faire l'objet d'une ACS, puis d'une ACC avec Franck. Pascal est en fin de carrière, il a environ 10 ans d'expérience sur le poste au début de l'intervention, et a travaillé autant d'années comme éboueur dans le service. Avant les analyses en autoconfrontations simple et croisées, et dans un discours général sur l'objet (les plaintes) retenu pour celles-ci, Pascal affirme ne pas traiter les plaintes au motif que cela ne serait pas à lui de le faire. Sur un plan administratif, il avance que sa hiérarchie a toutes les données pour répondre aux plaignants, si elle reprend les renseignements transmis quotidiennement et par plusieurs canaux par les chefs d'équipe. Il soutient aussi que les plaintes sont le plus souvent injustifiées, car relevant de malpropretés dont les usagers seraient responsables (liées à des dépôts hors containers des déchets ou encore à des incivilités). Il met enfin fortement les plaintes en lien avec un manque de moyens du service dont, là encore, il ne se considère pas responsable. Pour cet ensemble de raisons, le traitement des plaintes sur le terrain, avec des actions correctives en urgence, lui semble inutile et/ou illégitime. Les observations de son activité en atelier, mais surtout le travail en autoconfrontation, vont largement complexifier ce premier discours comme nous allons le voir dans l'analyse réalisée avec Pascal concernant cette plainte du 111 de l'avenue du Layon.

\subsubsection{L'autoconfrontation simple avec Pascal}

Cette ACS a lieu dans l'atelier de Pascal et porte sur un formulaire reprenant la plainte, envoyé à Pascal par sa hiérarchie pour qu'il la traite, avec notamment des cases à renseigner. Aucune de ces cases n'a été remplie par Pascal. Pascal n'a pas non plus de traces dans ces mails d'un retour qu'il aurait pu faire à sa hiérarchie, hors formulaire, sur le sujet. Le dialogue s'engage sur ce qui paraît être un non-traitement de la plainte, en tout cas au plan administratif, et est interrompu par un appel d'une stagiaire de sa division lui indiquant qu'un de ses engins de lavage a été supprimé la veille. Cet engin devait justement nettoyer l'avenue du Layon. La discussion sur la plainte reprend suite à cet appel, enrichie par cette nouvelle information. Le premier extrait ci-dessous correspond à ce moment-là. 
Tableau 1 : Extrait de l'autoconfrontation simple avec Pascal. Table 1: Extract from simple self-confrontation with Pascal

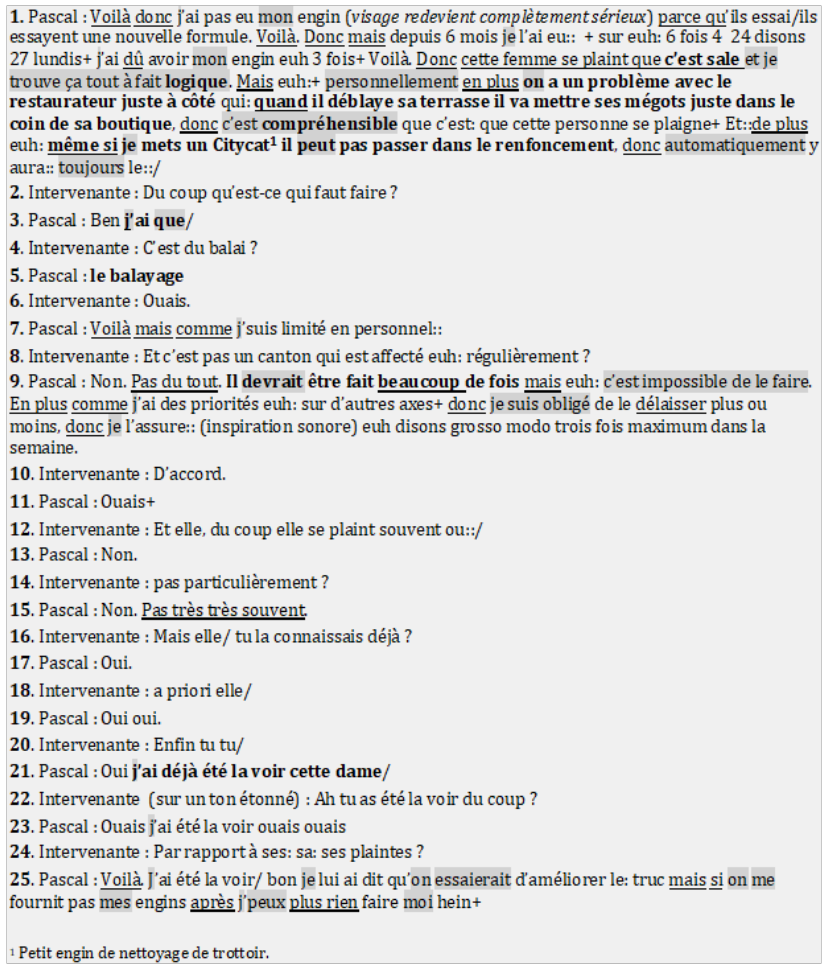

Contrairement à ce qu'il dit le plus souvent sur les plaintes, Pascal dit considérer cette plainte «justifiée », «logique », " compréhensible ». Dans le dialogue avec l'intervenante, il explique la malpropreté de cette zone par sa situation géographique (tour de parole 1, désormais TDP 1 : elle est dans un renfoncement, à côté d'un restaurateur qui balaye sa terrasse en poussant les mégots devant la boutique) et par un manque de moyens humains et techniques (TDP 1 à 9 : le renfoncement fait qu'on ne peut pas faire passer d'engin de nettoyage de trottoir, il manque de personnel pour assurer un balayage régulier de la zone, la laveuse avec lancier qui nettoie normalement l'avenue tous les lundis est régulièrement supprimée $\left.{ }^{8}\right)$. Les propos qu'il tient dans ce cadre relèvent $d u$ vrai et du certain (les modalisations effectuées, comme par exemple avec les adverbes « automatiquement » ou "toujours » en 1, ou le commentaire réflexif «c'est impossible de le faire » en 9 relèvent de la certitude, de l'évidence ; le discours s'articule autour de liens de cause à effet explicites, avec notamment une répétition de « donc » et de "voilà » tout au long de l'extrait) et ses propos sont très personnalisés (avec des énoncés à la première personne du singulier en $1,3,7,9,21,23$ et 25 , ou encore des modalisations marquant une prise en charge personnelle du discours comme avec l'adverbe "personnellement» en 1 ou des appréciations subjectives et évaluatives marquées du type "je trouve ça tout à fait logique » en 1 ou "je suis obligé de délaisser [ce canton] » en 9) : il est très impliqué dans ce qu'il avance, et dans un rapport d'adhésion fort à ce qu'il dit.

Il donne par ailleurs à voir ce qu'il a pu mettre en place par rapport à cette plainte : il est allé voir la plaignante (TDP 21 et 23), dans la suite du dialogue il dira aussi être allé voir le restaurateur impliqué de son point de vue dans la malpropreté de la zone, en avoir parlé à ses supérieurs pour que celui-ci soit éventuellement verbalisé, et avoir même détourné parfois des balayeurs ou des engins affectés ailleurs pour venir 
nettoyer cette petite zone. Il nous montrera enfin un système d'archivage papier des plaintes qu'il a mis en place et qui lui permet de retrouver les plaintes reçues par son atelier et, entre autres, de voir si elles sont nouvelles ou récurrentes. Il a ainsi un historique de cette plainte sur lequel il s'appuie pour justifier son caractère répétitif. Lors de l'ACS, il décrit donc cette plainte comme étant justifiée du côté de la plaignante (elle a raison de se plaindre de la malpropreté devant son magasin qui est réelle et n'est pas de son fait), et problématique du côté du service dont il pointe la responsabilité (s'il y a des difficultés objectives à nettoyer la zone en raison de sa configuration, il y a aussi, selon lui, des insuffisances du service qui manque de personnel et n'attribue pas les engins de nettoyage comme il le devrait).

Derrière un discours premier qui affirme qu'il ne se mobilise pas dans l'activité de traitement de plainte, on voit donc que Pascal, pour une plainte en particulier, peut mettre un ensemble d'actions en place. Dans ce cas précis, si la plainte n'est pas traitée, c'est uniquement sur un plan administratif. S'il ne se mobilise pas à destination de ses supérieurs hiérarchiques en refusant de leur envoyer des éléments qui pourraient leur permettre de répondre à la plaignante, il se mobilise en revanche à destination de la plaignante et cherche à améliorer la propreté des abords de son magasin. Deux niveaux au moins peuvent alors être distingués :

- D'une part celui d'un désengagement dans le discours qui, particulièrement dans le cadre de l'intervention, peut s'entendre comme une forme de résistance à la prescription et une forme de revendication de son désaccord avec cette prescription de traiter toutes les plaintes, adressées à ses collègues (pairs et hiérarchiques). De son point de vue, cette prescription et les procédures qui l'accompagnent ne sont pas adaptées et masquent la réalité des problèmes rencontrés sur le terrain, notamment le fait que les ateliers n'ont pas les moyens de nettoyer correctement l'ensemble des zones dont ils ont la charge.

- D'autre part celui d'un engagement dans les actes, perceptible dans ce qu'il dit essayer de faire, mais aussi être empêché de faire, du fait des contraintes et du manque de ressources qu'il rencontre dans l'organisation du travail. Il exprime d'ailleurs une forme d'impuissance dans la situation (TDP 25) : "J'ai été voir [la plaignante], je lui ai dit qu'on essaierait d'améliorer le truc, mais si on ne me fournit pas mes engins après je peux plus rien faire moi ». On notera par ailleurs dans cette formulation l'utilisation de la négation " ne... plus », suggérant une dégradation de la situation dans le sens de la disparition de la possibilité de faire quelque chose qui existait avant.

\subsubsection{L'autoconfrontation croisée entre Pascal et Franck}

L'autoconfrontation croisée avec Franck est l'occasion de revenir sur le traitement de la plainte de l'avenue du Layon et de franchir un nouveau pas dans le travail de coanalyse. Les deux chefs d'équipe visionnent le film dans lequel Pascal reçoit cet appel de la stagiaire de sa division et le dialogue engagé alors avec l'intervenante. L'appel, et le silence qui suit cet appel sont en effet sur le film de l'ACS, et c'est sur cette même situation qu'un nouveau dialogue s'engage en ACC, entre collègues cette fois. Pascal est poussé dans son argumentation et ajoute de nouveaux éléments dans son discours. À cette occasion, il est aussi possible de voir, pour partie, ce qui lui est propre et ce qu'il partage avec son collègue dans ses façons de faire et de voir. Franck est chef d'équipe depuis 3 ans et c'est son premier poste dans le service, il est responsable d'un secteur de nettoyage limitrophe à celui de Pascal et chacun connaît bien la zone d'intervention de l'autre. 
Tableau 2 : Extrait de l'autoconfrontation croisée entre Pascal et Franck. Table 2: Extract from crossed self-confrontation between Pascal and Franck

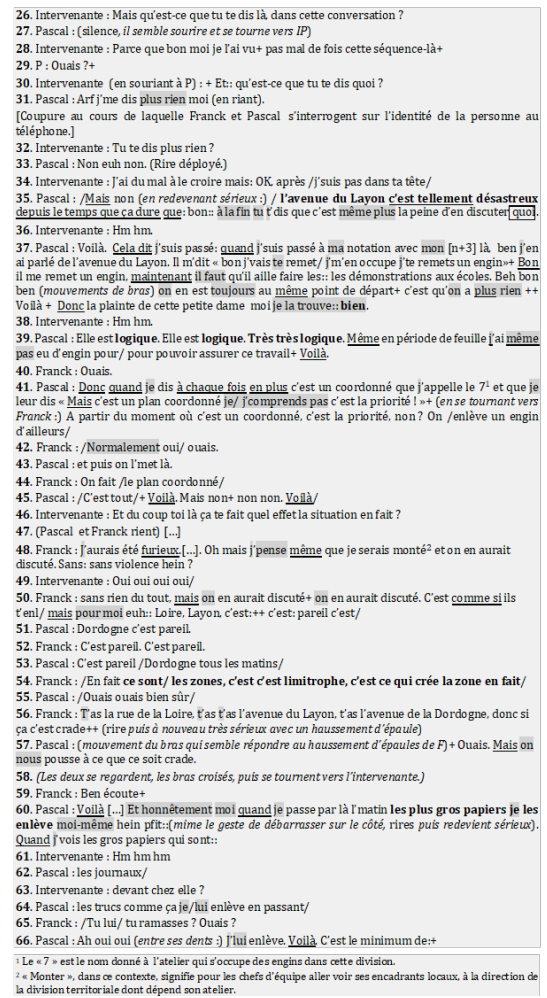

Dans cet extrait, nous revenons donc sur la conversation téléphonique qui a interrompu l'ACS, au cours de laquelle il était annoncé à Pascal qu'un engin avait été supprimé sur la zone concernée par la plainte de l'avenue du Layon. Dans cet échange, Pascal alterne le rire (TDP 31, 33, 47, 60) et le sérieux (TDP 35 à 45, TDP 51 à 66). Ses rires sont parfois très déployés, on serait tenté de parler de "rires francs ", dans le sens de rires auxquels on pourrait se fier comme exprimant une joie, un plaisir sans équivoque. Mais ramener à ce qui est dit qui ne prête pas à rire et au retour à la gravité qui suit (comme en 35 et 60), on est plutôt tenté de parler d'un « rire jaune » ou de rires défensifs. Les propos que finit par verbaliser Pascal sont en effet cinglants et sans appel quant à son évaluation de la situation de l'avenue du Layon et de ses effets sur lui : il ne se dit plus rien (TDP 31, 33,35) et n'en dit plus rien aux autres non plus, car «l'avenue du Layon c'est tellement désastreux depuis le temps que ça dure que bon à la fin tu t'dis que c'est même plus la peine d'en discuter quoi ».

On remarquera ici une forme d'effacement énonciatif avec l'utilisation d'un "tu » indéfini qui crée l'impression d'un point de vue générique et partagé. Pendant ces premiers tours de parole, son collègue reste silencieux, puis il acquiesce et on voit dans la suite du dialogue qu'ils partagent l'analyse du problème : la plainte est bien justifiée, et la suppression régulière d'un engin de lavage est problématique. Elle est problématique pour cette plainte, mais plus largement parce que cela empêche de faire le plan de nettoyage coordonné $e^{9}$ prévu, que Franck et Pascal s'accordent à dire prioritaire (TDP 41 et 42), ainsi que de nettoyer les rues limitrophes du secteur dont Pascal a la responsabilité (TDP 50 à 56). Nettoyer les rues limitrophes de son secteur, qui sont en général des grandes rues ou avenues assez passantes et qui «[créent] la zone» (TDP 54) à nettoyer, est d'un commun accord considéré central. De façon 
générale, à différents moments de cette ACC, on notera comme ici des moments de coénonciations entre Franck et Pascal, avec des analyses et évaluations partagées de la situation étudiée. Le caractère vrai ou indiscutable que prennent les énoncés de Pascal à certains moments, comme souligné plus haut dans l'analyse de l'ACS, tire ainsi peutêtre pour partie sa source de repères et pratiques professionnels partagés, construits, transmis et discutés collectivement, de l'ordre du genre professionnel, entendu comme un répertoire de manières d'agir et de penser, partagées dans un métier (Clot, 2008).

$\mathrm{Si}$, comme lors de l'ACS, Pascal adopte une posture assez assurée, et ce d'autant plus que Franck approuve régulièrement ses propos, on peut remarquer dans cet extrait des formes de modération dans son discours : "[il ne se dit] plus rien » et "c'est même plus la peine d'en parler ", "cela dit » (TDP 37) il « en a discuté » (TDP 37 et 41) dans au moins deux contextes. Mais ce qu'il relate, en s'appuyant sur des discours rapportés, contribue finalement à souligner une nouvelle fois sa difficulté à trouver dans l'organisation ce dont il aurait besoin pour nettoyer convenablement la zone. Dans un premier contexte (TDP 37), il rapporte sa sollicitation de son supérieur hiérarchique $(n+3)$ : il s'est saisi du cadre de son entretien annuel de notation pour souligner le problème de l'avenue du Layon, son encadrant a entendu sa demande, mais selon Pascal cela n'a eu que très partiellement des effets puisque les engins, au moment de l'ACS et de l'ACC, sont à nouveau mobilisés pour faire d'autres choses. Pascal formule alors à nouveau un point de vue à la fois catégorique et peu nuancé, très marqué subjectivement, dans des modalités de surénonciation (utilisation quasi exclusive de la première personne $\mathrm{du}$ singulier, liens logiques explicites, modalisation du propos spécifiant son point de vue propre et son adhésion à ce qu'il dit): «on a plus rien» (TDP 37), "la plainte de cette petite dame moi je la trouve bien" et "très très logique » (TDP 37 puis 39), « même en période de feuilles j'ai même pas eu d'engin pour pouvoir assurer ce travail » (TDP 39). Dans un second contexte (TDP 41), c'est auprès de l'atelier qui fournit les engins qu'il a tenté de plaider sa cause, sans résultat non plus.

$\mathrm{Au}$ final, les deux exemples ne viennent pas nuancer, mais plutôt corroborer l'idée que "c'est même plus la peine d'en discuter ", et donner un sens concret à la forme négative " plus »: il a bien essayé d'en discuter, mais cela s'est soldé par des échecs, il semble alors avoir fait l'expérience de l'inutilité de sa parole. On comprend alors mieux pourquoi ce ne serait plus la peine d'en discuter : dans son expérience, cela ne mène à rien. Au-delà de ce tour de parole, l'utilisation répétée des formulations négatives fortes et insistantes «plus rien» (TDP 31 et 37), et « même plus»/ « même pas» (TDP 35 et 39) donne l'impression qu'aucune chose, même petite ou considérée comme minimale par Pascal, ne serait possible dans la situation. Ces formulations peuvent aussi suggérer des possibilités qui ont existé et qui auraient totalement disparu, réduites à "rien ». Combinées avec des compléments circonstanciels et un adverbe modalisateur qui donnent des indications de temps et de fréquence allant dans le sens d'une répétition (TDP 35 : «depuis le temps que ça dure », TDP 37 : «toujours », TDP 41 : "à chaque fois»), les formulations de Pascal dessinent une situation qui n'avance pas: "On en est toujours au même point de départ » (TDP 37).

Il se dégage de la séquence l'impression d'une usure et d'une lassitude qui s'installeraient ou se seraient installées chez Pascal. Au désengagement revendicatif et à son engagement en acte vient finalement s'ajouter un désengagement de l'ordre du renoncement, conséquence d'un engagement qui aurait connu de façon répétée l'échec. Ainsi, à son engagement insistant, mais infructueux, Pascal tente peut-être de 
substituer une forme de désengagement défensif susceptible de le préserver de l'échec répété et de ses implications subjectives (comme la colère, la lassitude, ou l'usure). Ce désengagement est partiellement partagé avec Franck et semble presque inévitable : on rencontre un accord tacite entre eux sur le fait qu'« on les pousse à ce que ce soit crade » (TDP 57 à 60) et qu'il n'y aurait en quelque sorte rien à faire à cela. Mais on voit aussi dans les échanges que Franck semble prêt à continuer à réagir, à ne pas retenir sa «fureur » (TDP 48 et 50), et à tenter agir dans le type de situation décrite par Pascal (ici en tentant une nouvelle discussion, au moment où le problème survient, avec ses encadrants locaux).

Plus loin dans l'extrait est à nouveau visible la particularité de ce que Pascal fait pour répondre à cette plainte: tous les matins, en allant travailler, il passe devant la boutique et ramasse les gros papiers accumulés devant. L'activité de Pascal concernant cette plainte prend alors une épaisseur supplémentaire. La modalisation de son propos (avec le modalisateur "honnêtement" en 60 qui nous place dans le registre d'une déclaration franche et sincère), ainsi que l'insistance sur son implication dans ce qui est fait (mobilisation du «je » en 60, 64 et 66, renforcée par l'utilisation de «moi » et « moimême » en 60 aussi) nous semblent traduire une forme d'adhésion et de validation fortes de ce qu'il dit: cette pratique est importante pour lui et constitue un quasidevoir. "C'est le minimum », comme il le dit à la fin de l'extrait en 66. Cette pratique est par ailleurs très singulière. Quand il ramasse les papiers en allant travailler, cela veut dire qu'il participe directement au travail de propreté, et cette confrontation directe au déchet n'a rien d'anodin (Lhuilier, 2005 ; 2011). C'est loin d'être le cas de tous les chefs : certains nous ont semblé toujours faire ramasser et ne jamais ramasser eux-mêmes. Et dans le cas présent, Pascal le fait dans un contact direct avec les déchets (sans pelle, sans balai, sans gants, contrairement aux agents qui, plus tard dans la matinée, interviendront "équipés » sur le terrain). Ajoutons que ceci se passe en dehors du temps de travail, quand il passe le matin avenue du Layon pour se rendre à son atelier. Ce triple choix - faire soi-même régulièrement, sans l'intermédiaire d'équipements, et en dehors des heures de travail - traduit une nouvelle fois la particularité d'un engagement fort et singulier que nous n'avons pas rencontré, en tout cas pas dans cette proportion et sous cette forme très personnelle, dans ce à quoi nous avons eu accès lors des co-analyses avec les autres chefs d'équipe participant à notre intervention.

\section{Une approche clinique de l'engagement et du désengagement}

\section{1. Étudier la complexité d'un processus pour en comprendre les ressorts}

Nous avons cherché dans cet article à analyser la singularité, la particularité de l'engagement/désengagement, pour dessiner la complexité d'un processus qui est peu envisagé comme tel dans la littérature sur cette question. L'analyse d'une subjectivité singulière, et même inattendue, déployée dans une activité professionnelle nous permet d'avancer des dynamiques, des aspérités, des conflictualités de l'engagement que les perspectives citées en amont du texte - celles que nous avons nommées «l'engagement comme lien à une cible » ou « l'engagement comme état»-permettent peu d'envisager. 

un professionnel peut se mobiliser de façon différenciée selon les situations et les destinataires de son activité. Ici, la désinvolture et le désengagement affichés dans le discours initial laissent rapidement la place à une réalité beaucoup plus contrastée : Pascal peut être très mobilisé dans la réalisation de certaines tâches s'il considère que la situation le justifie, et vis-à-vis de certains destinataires dont il comprend et reconnaît comme légitimes les demandes et les attentes. Concernant cette plainte de l'avenue du Layon, dans ce qu'il dit, dans les dialogues, de ce qu'il fait, le discours d'inactivité affichée et assumée se fissure pour laisser voir une activité qui, pour une part, va même bien au-delà du prescrit et de ce que ses collègues font. Il traite notamment très activement et durablement la plainte sur le terrain.

作 qu'il traduisait à la fois une forme de résistance, voire de riposte, et une forme de défense. Au cours de cette intervention, Pascal et ses collègues ont régulièrement pointé des moyens insuffisants, selon eux, et le problème de leurs demandes laissées sans réponse. Dire qu'on ne fait pas, ou refuser de faire, comme le fait Pascal, peut être vu comme une façon de résister, de ne pas se soumettre silencieusement à des règles ou des ordres qui leur font prendre en charge les conséquences de problèmes relevant pour eux de dysfonctionnements organisationnels. L'afficher auprès de nous et dans le cadre de l'intervention est peut-être même un moyen de transformer la résistance silencieuse en revendication ouverte. Mais le désengagement, dans une situation comme celle analysée ici, peut aussi être une façon de tenter de se défendre des effets subjectifs que peut avoir la prise en charge de certaines plaintes, quand elle renvoie à des problèmes structurels et des échecs répétés. Nous l'avons vu, Pascal peut s'impliquer beaucoup et très personnellement vis-à-vis des usagers et dans le traitement de certaines plaintes, mais il y fait l'expérience de la confrontation à des obstacles récurrents, de l'impossibilité d'agir, de la parole inutile, etc. Tout cela a des conséquences subjectives coûteuses et, sous cet angle, s'engager n'est pas toujours synonyme de santé.

51 L'analyse détaillée d'un autre cas (Sandoval \& Kostulski, 2021), dans cette même visée de comprendre la complexité des processus d'engagement et de désengagement, a permis de mettre au jour une autre forme d'engagement conflictuel tout aussi singulière et inattendue, chez une collègue de Pascal. Nous mettons en évidence dans cette autre configuration une dimension transgressive au plan de l'action sur le terrain (réaliser et faire réaliser des actions interdites pour rester efficace dans son travail), assortie d'une conformité aux règles de l'organisation au plan du reporting et des procédures en interne par rapport à la hiérarchie (comme solliciter le service dédié, rendre compte, alerter, remplir les documents attendus... en sachant que la réponse organisationnelle n'arrivera pas). Dans cet exemple, comme dans celui de Pascal, la configuration d'engagement/désengagement identifiée à des effets subjectifs forts, et se pose la question de ses effets sur la santé. Ainsi, pour Pascal comme pour d'autres professionnels, la force de leur engagement peut en quelque sorte se retourner contre eux et nourrir des formes d'amertume, d'isolement, de déception, ou d'épuisement, délétères pour leur santé. Se désengager devient alors potentiellement une façon de se protéger et de se préserver, et cette forme de désengagement peut se comprendre comme la conséquence d'un engagement durablement infructueux. 
52 Au-delà de la situation particulièrement complexe et conflictuelle de Pascal, des formes de désengagement, qui relèvent à la fois de la résistance et de la défense, se sont montrées souvent présentes chez les chefs d'équipe. L'analyse des dialogues avec Pascal permet finalement d'identifier des mouvements et conflits d'engagement/ désengagement que nous avons repérés chez nombre de ses collègues, mais qui prennent chez lui une forme extrême et singulière du fait de la conflictualité paroxystique à laquelle ils mènent. L'analyse de ces mouvements et conflits, de par leurs effets sur la santé et sur l'efficacité des professionnels, mais aussi de par leur caractère collectif, mériterait d'être approfondie. Les échanges dans nos collectifs de chefs d'équipe, mais aussi le temps passé dans les ateliers auprès de chefs d'équipe novices, nous conduisent à faire l'hypothèse que, si ces formes de désengagement se rattachent à des expériences vécues de façon répétée par ces professionnels, elles se transmettent aussi entre eux comme des façons de faire possibles et même nécessaires dans le métier. Les impossibilités intériorisées par les plus anciens, l'idée qu'il vaudrait mieux ne pas trop en faire, seraient en quelque sorte intégrées au "genre professionnel » des chefs d'équipe, en lien avec l'expérience qu'ont les plus anciens de l'organisation dans laquelle ils travaillent. Dans ce milieu professionnel qui œuvre à la collecte des déchets, cette dimension collective du désengagement pourrait peut-être aussi se comprendre comme le refus ou la difficulté de s'engager dans des tâches socialement dévalorisées, car relevant du « sale boulot » (Lhuilier, 2005). Allant dans ce sens, lors de nos premiers pas sur ce terrain, il a pu nous arriver d'être confrontées à des chefs d'équipe qui minimisaient ou discréditaient a priori l'engagement qu'ils auraient pu mettre dans la réalisation d'une besogne qu'ils disaient être simple, peu intéressante et ne demander aucune connaissance ou compétence particulière. Pour autant, les analyses de l'activité menées avec les participants à notre intervention révèlent que malgré ces discours premiers discréditant souvent leur métier, les chefs d'équipe montrent un engagement fort qui dépasse les possibilités offertes par l'organisation du travail. Les liens entre les formes conflictuelles d'engagement/ désengagement et le "sale boulot" seraient ainsi une voie à explorer, mais sans prendre « au pied de la lettre » ce que les professionnels disent a priori.

\subsection{Engagement et horizon de l'intervention}

53 Cette intervention-recherche peut mener à une question : devons-nous chercher à agir directement sur l'engagement au travail dans nos interventions? Dans ce cas précis, la commande n'était pas de ce côté et ce n'est généralement pas la façon que nous avons de prendre, en clinique de l'activité, les commandes et demandes qui nous sont adressées. Pour rappel, il s'agit pour nous de créer des dispositifs qui visent des possibilités de développement de l'efficacité des professionnels dans leur activité, efficacité entendue, à partir des propositions d'Yves Clot $(2004 ; 2008)$, comme une conjonction entre d'une part l'efficience du geste du professionnel et d'autre part le sens que ce geste va prendre pour lui. Ce développement de l'efficacité est central dans notre action, car il va de pair avec le développement de la santé : la santé dépend de la possibilité de se sentir efficace ou, pour le dire dans les termes de la clinique de l'activité, de la possibilité de se reconnaître dans ce que l'on fait. Notre travail sur le terrain, le travail clinique avec les professionnels comme les dialogues engagés avec la hiérarchie et le comité de pilotage, s'est inscrit dans cet horizon, mais s'est confronté de manière récurrente à des formes d'engagement et de désengagement le rendant 
difficile, tant du côté de l'engagement au travail dans l'activité quotidienne des professionnels que de l'engagement de l'ensemble des professionnels dans l'intervention elle-même.

Au plan de la recherche, c'est l'expérience de cette confrontation récurrente à des formes très particulières ou déroutantes d'engagement/désengagement qui nous a menées à tenter de mieux comprendre les dynamiques subjectives de l'engagement et $\mathrm{du}$ désengagement, au regard des multiples conflictualités que ces dynamiques présentaient sur le terrain et au sein même de cette institution. Nous n'avons pas identifié une forme générale d'engagement ou de désengagement qui pourrait qualifier un métier ou une organisation, mais plutôt donc des configurations conflictuelles multiples déclinant des formes subjectives d'engagement contrarié. Se pose alors la question des liens possibles entre le modèle de l'organisation bureaucratique que nous avons rencontré et des formes d'engagement/désengagement qui peuvent mener in fine à de l'inefficacité, de l'usure, ou au développement de formes inattendues, subversives ou transgressives, de mobilisation dans l'activité (Sandoval \& Kostulski, à paraître). Cette investigation des rapports entre modèle organisationnel et dynamiques d'engagement/ désengagement serait intéressante à développer sur ce terrain et dans d'autres interventions, pour tenter de mieux saisir avec les acteurs de terrain comment transformer des organisations qui finissent par être inefficaces, décourager les initiatives et démobiliser les professionnels.

Si nous n'avons pas cherché, en première instance, à développer de l'engagement au travail, notre intervention a eu, dans les faits, un effet d'engagement ou de réengagement pour une partie des professionnels avec lesquels nous avons travaillés. Les échanges autour des co-analyses réalisées ont en effet amené la hiérarchie de la division à prendre en compte le point de vue des chefs d'équipe. Les responsables de cette division ont revu le circuit de traitement de la plainte, en s'appuyant sur différentes réunions ayant permis aux chefs d'équipe de partager leurs analyses avec d'autres échelons et fonctions concernés eux aussi par le traitement des plaintes, et, en retour, d'entendre les contraintes de ces autres acteurs pour proposer avec eux des compromis acceptables. Un nouveau circuit, cherchant à assurer une meilleure circulation de l'information et aussi une meilleure répartition des tâches au sein de la division, a été proposé. Le traitement administratif de la plainte a été reporté principalement vers les encadrants des chefs d'équipe, les chefs d'équipe se concentrant sur leur traitement technique sur le terrain. Les outils de suivi de l'activité ont aussi été simplifiés et repensés afin de permettre une transmission plus aisée, plus fiable et plus directement partagée des nettoyages et lavages effectués sur le terrain. Après un temps d'expérimentation, ces changements ont été pérennisés. Les chefs d'équipe ont alors fait l'expérience de la possibilité, à laquelle beaucoup ne croyaient plus, d'agir sur l'organisation de leur service et d'avoir avec leurs encadrants des échanges argumentés et constructifs autour de leurs difficultés réelles. Là où le travail entre échelons semblait devenu rare et vite reproduire le même type de blocages (avec des problèmes qui remontent, des solutions qui redescendent, mais pas de construction collective et concertée de ces problèmes et de ces solutions), les participants à l'intervention ont pu expérimenter des cadres de «travail sur le travail » dans lesquels leurs analyses pouvaient se développer dans le dialogue avec celles des autres, et donner lieu à des transformations certes circonscrites, mais fructueuses. Se mobiliser et travailler avec ses pairs et ses encadrants face aux obstacles rencontrés est alors 
(re)devenu pour certains une alternative possible. Si certains professionnels ont tenté de maintenir ces cadres de "travail sur le travail ", ceux-ci étaient fragiles et nous n'avons que peu de visibilité sur le succès et la pérennisation de ces tentatives, notamment du fait de l'impossibilité de revenir sur ce terrain depuis le début de la pandémie et du changement de postes de nombre de nos interlocuteurs initiaux.

Notons un fait important selon nous : c'est finalement la prise pour objet et la mise au travail de l'activité réelle, y compris ses échecs et ses difficultés, qui vient soutenir voire développer -, l'engagement des professionnels, notamment en permettant de mettre au travail le sens et l'efficience des gestes professionnels du quotidien. À l'inverse, on peut faire l'hypothèse que quand l'organisation du travail n'offre plus de possibilités aux professionnels de se confronter collectivement aux problèmes rencontrés, elles participent à produire du désengagement et de l'absentéisme. Voilà une piste qu'il faudrait développer et que nous avons tenté de mettre au travail avec nos commanditaires, en leur proposant de considérer l'absentéisme comme une conséquence de l'impossibilité pour les professionnels de pouvoir faire avancer les problèmes du travail. En outre, il serait intéressant de regarder dans quelle mesure des mouvements de réengagement sont possibles, au regard de l'intensité du désengagement individuel et collectif qui s'est développé. Les difficultés à mobiliser des professionnels pour participer au cadre de l'intervention peuvent suggérer que le type de travail collectif que nous proposions ne faisait, pour certains, plus partie d'un « champ des possibles » dans leur activité (Haué, Le Bellu \& Barbier, 2020), rendant leur engagement dans l'intervention et leur réengagement dans l'activité difficile.

\section{Pour conclure}

Nos analyses montrent que l'engagement et le désengagement peuvent se concevoir comme des mobilisations variables des professionnels dans la réalisation de leur activité, qui peuvent changer selon les situations et les moments, en fonction de ce qu'il est demandé de faire, des destinataires de l'activité, des moyens et ressources que les professionnels ont ou parviennent à construire pour faire ce qui est à faire, et des obstacles et contraintes qu'ils peuvent au contraire rencontrer, parfois de façon récurrente. L'engagement et le désengagement sont ainsi très liés à l'activité des professionnels, à ce qu'ils parviennent ou non à faire, et au sens que cela prend pour eux. Mais ce sens, comme les obstacles et ressources rencontrés, ne peuvent se saisir et se comprendre qu'en analysant précisément cette activité avec les professionnels. Ainsi, s'engager et se désengager peuvent permettre de s'adapter, de se défendre, de résister dans la situation, ou encore d'essayer d'agir sur celle-ci. Dans l'exemple que nous avons retenu, coexistent ainsi : une forme de désengagement de l'ordre de la revendication et de la résistance; un engagement fort et très personnel dans la réalisation d'une activité analysée comme nécessaire et importante par le professionnel; et un désengagement plus défensif, pour une part partagé, dont nous avons fait l'hypothèse qu'il pouvait être une façon de se protéger des échecs répétés.

Notre cheminement nous mène à cette conclusion. La question de l'engagement devient plus complexe lorsqu'elle est explorée dans ses liens avec les différentes dimensions de l'activité quotidienne. L'engagement et le désengagement se développent et s'infléchissent dans le cours de l'activité, dont ils peuvent être des modalités, des sources, et des effets, voire des visées, dans des rapports de co-engendrement. Ils sont 
alors des processus qui ne sont pas exclusifs l'un de l'autre; ils peuvent être concomitants et sont potentiellement conflictuels ou ambivalents. Dans la situation analysée ici, continuer à s'engager dans le traitement des plaintes peut aisément coexister avec l'idée qu'il serait mieux de ne plus le faire. Comprendre l'engagement et le désengagement ne peut ainsi faire l'économie d'une analyse de l'activité et des impasses de cette activité, l'engagement comme le désengagement se montrant relever ni des attributs de la personne, ni des attributs d'une situation, mais bien de la rencontre entre les deux au cours de l'activité réelle.

Nous avons tenté de mettre en évidence cette complexité en mobilisant des éléments de la situation de travail, mais d'autres seraient à explorer, comme ceux relevant des autres milieux de vie de sujet. Dans son étude de l'absentéisme, Veil (2012) invitait déjà à comprendre les conduites dites inadaptées au travail comme relevant d'une " inadaptation subjective et relative", "fruit de conflits non résolus qui concernent soit la vie au travail, soit la vie hors travail, soit l'articulation de ces deux champs » (p.166). Une autre poursuite possible de ce travail de recherche serait alors d'investiguer les conduites d'engagement et de désengagement au travail, en les mettant aussi en rapport avec ce qui peut se jouer dans les autres domaines de la vie du sujet, avec les contraintes et les ressources issues de ces autres sphères (Almudever, Martin, \& Croity-Belz, 2016 ; Curie, \& Hajjar, 1987), dans une dynamique subjective plus globale.

\section{BIBLIOGRAPHIE}

Almudever,B., Martin, A.-C., \& Croity-Belz, S. (2016). Rapports travail/hors travail. In G. Valléry, M.-É. Bobillier Chaumon, É. Brangier, \& M. Dubois (Eds.), Psychologie du travail et des organisations : 110 notions clés (p. 349-353). Dunod.

Bakker, A.B., \& Leiter M.P. (Eds.). (2010). Work engagement: A handbook of essential theory and research. Psychology Press.

Bakker, A. B., Schaufeli, W. B., Leiter, M. P., \& Taris, T. W. (2008). Work Engagement: An Emerging Concept in Occupational Health Psychology. Work \& Stress, 22, 187-200. http://dx.doi.org/ $10.1080 / 02678370802393649$

Bonnemain, A. (2015). Les paradoxes de l'intensité affective dans l'autoconfrontation: L'exemple de l'activité dialogique des chefs d'équipe de la propreté de Paris (Thèse de doctorat, Cnam, Paris, France). Repéré à : https://tel.archives-ouvertes.fr/tel-01344642/document

Bonnemain, A., \& Clot, Y. (2017). Clinique de l'activité : les affects dans l'autoconfrontation. In M. Santiago-Delefosse, \& M. Del Rio Carral (Eds.), Les méthodes qualitatives en psychologie et sciences humaines de la santé (p. 131-151). Dunod.

Clot, Y. (2004 [1999]). La fonction psychologique du travail (4édition corrigée). Presses universitaires de France.

Clot, Y. (2008). Travail et pouvoir d'agir. Presses universitaires de France. 
Clot, Y., Faïta, D., Fernandez, G., \& Scheller, L. (2000). Entretiens en autoconfrontation croisée : une méthode en clinique de l'activité. Perspectives interdisciplinaires sur le travail et la santé [En ligne], 2-1. http://dx.doi.org/10.4000/pistes.3833

Clot, Y., \& Quillerou-Grivot (2014). Qualité du travail : un problème avant d'être une solution. In D. Lhuilier (Ed.), Qualité du travail, qualité au travail (p. 141-159). Octarès.

Curie, J., \& Hajjar, V. (1987). Vie de travail, vie hors travail : la vie en temps partagé. In C. LévyLeboyer, \& J.-C. Spérandio (Eds.), Traité de psychologie du travail (p. 33-55). Presses universitaires de France.

Davezies, P. (1994). Position du médecin du travail face aux dimensions cognitives, psychiques et relationnelles du travail. Repéré à : http://philippe.davezies.free.fr/download/down/ Le_medecin_du_travail_face_aux_dimensions_cognitives_psychiques_relationnelles_1994.pdf [Paru en 1995 dans Archives des Maladies Professionnelles, 56, $\mathrm{n}^{\circ}$ 4, 294-306].

Dejours, C. (1998). Souffrance en France. La banalisation de l'injustice sociale. Seuil.

Dejours, C. (2004). Activisme professionnel : masochisme, compulsivité ou aliénation ? Travailler, $n^{\circ} 11,25-40$.

Desrumaux, P., Léoni, V., Bernaud, J., \& Defrancq, C. (2012). Les comportements pro- et antisociaux au travail : une recherche exploratoire testant deux échelles de mesure et leurs liens avec des inducteurs organisationnels et individuels. Le Travail Humain, 75(1), 55-87. http:// dx.doi.org/10.3917/th.751.0055

Duarte, A. (2017). Défenses et résistance en psychodynamique du travail (Thèse de doctorat inédite, Université Paris Descartes, France).

Efros, D., \& Schwartz, Y. (2009). Résistances, transgressions et transformations : l'impossible invivable dans les situations de travail. Nouvelle revue de psychosociologie, 7(1), 33-48. http:// dx.doi.org/10.3917

Falzon, P. (Ed.). (2004). Ergonomie. Presses universitaires de France.

Falzon, P. (Ed.). (2013). Ergonomie constructive. Presses universitaires de France.

Haué, J.B., Le Bellu, S., \& Barbier C. (2020). Le véhicule autonome : se désengager et se réengager dans la conduite, Activités [Online], 17(1). https://doi.org/10.4000/activites.4987

Kahn, W. A. (1990). Psychological conditions of personal engagement and disengagement at work. Academy of Management Journal, 33(4), 692-724. Repéré à : http://dx.doi.org/10.2307/256287

Kerbrat-Orecchioni, C. (2009 [1980]). L'Énonciation. De la subjectivité dans le langage (4éd.). Armand Colin.

Klein H.J., Molloy J.C., \& Brinsfield, C.T. (2012), Reconceptualizing workplace commitment to redress a stretched construct : Revisiting assumptions and removing confounds. Academy of Management Review, 37(1), 130-151.

Kostulski, K. (2010). Quelles connaissances pour l'action en clinique du travail ? Une contribution à la question des rapports entre les actions de transformation et la production de connaissances en clinique du travail. In Y. Clot, \& D. Lhuilier (Eds.), Agir en clinique du travail (p. 27-38). Érès.

Kostulski, K. (2011). Formes et fonctions psychologiques des réalisations langagières : vers une psychologie concrète du langage (HDR, Université Paris 8, Saint-Denis, France).

Leplat, J., \& Hoc, J. M. (1983). Tâche et activité dans l'analyse psychologique des situations. Cahiers de psychologie cognitive, 3/1, 49-63. 
Lhuilier, D. (2005). Le « sale boulot ». Travailler, 14, 73-98.

Lhuilier, D. (2006). Cliniques du travail. Érès.

Lhuilier, D. (2011). Souillure et transgression : le travail sur le négatif psychosocial. In D. Corteel (Ed.), Les travailleurs des déchets (p. 33-43). Érès.

Lhuilier, D., \& Roche, P. (2009). Introduction « La résistance créatrice ». Nouvelle revue de psychosociologie, 7(1), 7-18.

Louche, C. (2017). Psychologie sociale des organisations (4 éd.). Armand Colin.

Maslach, C., \& Leiter, M.P. (1997/2011). Burn-out. Des solutions pour se protéger et pour agir. Les Arènes.

Meyer, J.P., \& Allen, N.J. (1991). A three-component conceptualization of organizational commitment. Human Resource Management Review, $\mathrm{n}^{\circ}$ 1, 61-89.

Meyer, J.P., \& Herscovitch, L. (2001). Commitment in the workplace: toward a general model. Human resource management review, 11(3), 299-326.

Molinier, P. (2006). Les enjeux psychiques du travail. Petite bibliothèque Payot.

Mowday R.T., Porter L.W., \& Steers R.M. (1982). Employee-organization linkages: The psychology of commitment, absenteeism and turnover. Academic Press.

Paillé, P. (2002). L'engagement normatif : essai de reconstruction. Psychologie du Travail et des Organisations, 8(3), 69-96.

Paillé, P. (2005). Engagement organisationnel et modes d'identification. Dimensions conceptuelle et empirique. Bulletin de psychologie, 58-6(480), 705-711.

Paillé, P. (2009). Engagement et citoyenneté en contexte organisationnel : un examen empirique sur l'apport des cibles multiples d'engagement à la prédiction des comportements de citoyenneté organisationnelle. Le Travail Humain, 72(2), 185-204. http://dx.doi.org/10.3917/th.722.0185

Paillé, P. (2012). Changement organisationnel, résistance et engagement des salariés. Psychologie du Travail et des Organisations, 18(1), 61-80.

Rabatel, A. (2008). Homo narrans. Pour une analyse énonciative et interactionnelle du récit. Lambert Lucas.

Rabatel, A. (2014, octobre). Point de vue, empathie, prise en charge. Conférence, Universidade Federal do Rio Grande do Norte, Natal, Brésil.

Roche, P. (2016). La puissance d'agir au travail. Recherches et interventions cliniques. Érès.

Sandoval, A. (2020). Les dynamiques d'engagement et de désengagement au travail. Contribution à leur conceptualisation en clinique du travail, à partir d'une intervention dans un service public de la propreté, (Thèse de doctorat, Cnam - Hesam Université, Paris, France). Repéré à : https://tel.archivesouvertes.fr/tel-03151307/document

Sandoval, A., \& Kostulski, K. (2021). « Malheureusement on a une conscience... » : l'engagement des professionnels, ressource ou obstacle pour la santé ? Bulletin de psychologie, 2(2), 105-116.

Sandoval, A., \& Kostulski, K. (à paraître). Transformer le travail dans une organisation bureaucratique ? L'exemple d'une intervention dans le service de la propreté d'une collectivité territoriale. Revue des conditions de travail.

Schaufeli, W.B. (2013). What is engagement? In C. Truss, K. Alfes, R. Delbridge, A. Shantz, \& E. Soane (Eds.), Employee Engagement in Theory and Practice (p. 15-35). Routledge. 
Schaufeli, W. B., \& Bakker, A. (2010). Defining and measuring work engagement: bringing clarity to the concept. In A. B. Bakker, \& M. P. Leiter (Eds.), Work engagement: A handbook of essential theory and research (p. 10-24). Psychology Press.

Schaufeli, W.B., Bakker, A., \& Salanova, M. (2006). The Measurement of Work Engagement With a Short Questionnaire. A Cross-National Study. Educational and Psychological Measurement, 66(4), 701-716.

Scott, J. (1990/2006). Infra-politique des groupes subalternes. Vacarme, 36(3), 25-29. http:// dx.doi.org/10.3917/vaca.036.0025

Solinger, O. N., van Olffen, W., \& Roe, R. A. (2008). Beyond the three-component model of organizational commitment. Journal of Applied Psychology, 93(1), 70-83. http://dx.doi.org/ 10.1037/0021-9010.93.1.70

Vandenberghe, C. (2016). L'engagement organisationnel. In G. Valléry, M.-É. Bobillier Chaumon, É. Brangier, \& M. Dubois (Eds.). Psychologie du Travail et des Organisations : 110 notions clefs (p. 175-178). Dunod.

Veil, C. (2012). Vulnérabilités au travail. Toulouse, France : Érès.

Vion, R. (2004). Modalités, modalisations et discours représentés. Langages, n 156, 96-110.

Vion, R. (2005). Modalités, modalisations, interaction et dialogisme. In J. Bres (Ed.), Dialogisme et polyphonie. Approches linguistiques (p. 143-156). De Boeck Supérieur.

Vion, R. (2011). La modalisation. Un mode paradoxal de prise en charge. In P. Dendale (Ed.), La prise en charge énonciative : études théoriques et empiriques (p. 75-91). De Boeck Supérieur.

Viviers, S. (2014). Souffrance identitaire de métier. Des conseillères et des conseillers d'orientation s'interrogent sur le présent et l'avenir de leur profession en milieu scolaire (Thèse de doctorat, Université de Laval, Québec, Canada). Repéré à : http://www.crievat.fse.ulaval.ca/fichiers/ site_crievat/documents/Essai_memoire_these/2014-2015/

Viviers_2014_Souffrance_identitaire_de_metier.pdf

\section{ANNEXES}




\section{Annexe 1. Grille récapitulative des dimensions et indices retenus pour les analyses langagières. Summary grid showing the dimensions and indicators selected for the language analyses}

\begin{tabular}{|c|c|c|c|}
\hline $\begin{array}{l}\text { Réalisation } \\
\text { langagière } \\
\text { considérée }\end{array}$ & $\begin{array}{l}\text { Dimensions } \\
\text { retenues }\end{array}$ & \begin{tabular}{|l|} 
Phénomènes \\
énonciatifs liés \\
aux \\
dimensions \\
retenues \\
\end{tabular} & $\begin{array}{l}\text { Exemples d'indices permettant d'appréhender les } \\
\text { dimensions et phénomènes énonciatifs retenus } \\
\text { pour l'analyse (Kerbrat-Orecchioni, 2009; Rabatel, } \\
\text { 2004, 2008, 2014; Vion, 2004, 2005, 2011) }\end{array}$ \\
\hline \multirow{2}{*}{$\begin{array}{l}\text { Énonciation } \\
\text { - contenu } \\
\text { propositionnel } \\
\text { - prise de } \\
\text { position par } \\
\text { rapport à ce } \\
\text { contenu }\end{array}$} & $\begin{array}{l}\text { Implication } \\
\text { (inscription ou } \\
\text { présence } \\
\text { subjective dans } \\
\text { l'énoncé) }\end{array}$ & 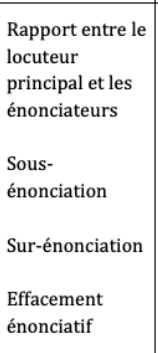 & $\begin{array}{l}\text { Déictiques personnels : pronoms personnels, } \\
\text { possessifs, démonstratifs } \\
\text { Déictiques spatiaux et temporels : ici, maintenant, } \\
\text { aujourd'hui, hier... } \\
\text { Recours à un énonciateur générique ou anonyme } \\
\text { Énoncés génériques, stéréotypiques ou doxiques } \\
\text { Degré de complexité des phrases } \\
\text { Choix lexicaux } \\
\text { Orientation discursive de l'énoncé : description, } \\
\text { explication, argumentation... } \\
\text { Comportements prosodiques et co-verbaux : } \\
\text { intonation, débit, gestes... } \\
\text { Syncrétisme entre locuteur et énonciateur }\end{array}$ \\
\hline & $\begin{array}{l}\text { Positionnement } \\
\text { (position } \\
\text { subjective par } \\
\text { rapport au } \\
\text { contenu l'énoncé : } \\
\text { adhésion, } \\
\text { degré d'accord, } \\
\text { appréciation...) }\end{array}$ & $\begin{array}{l}\begin{array}{l}\text { Modalités de } \\
\text { l'énoncé }\end{array} \\
\text { Modalisations } \\
\text { Commentaire } \\
\text { réflexif }\end{array}$ & $\begin{array}{l}\text { Modalisateurs : adverbe et locutions adverbiales } \\
\text { renvoyant à des appréciations, à des } \\
\text { évaluations (ex : certainement, éventuellement, } \\
\text { sans aucun doute...), } \\
\text { verbes subjectifs renvoyant à des perceptions et } \\
\text { des appréciations/évaluations (ex : aimer, préférer, } \\
\text { douter, craindre...), verbes de modalité (ex : } \\
\text { pouvoir, devoir, vouloir...), adjectifs appréciatifs et } \\
\text { évaluatifs (ex : évident, incertain, possible...), } \\
\text { certains substantifs ayant une valeur appréciative } \\
\text { ou évaluative, ... } \\
\text { Pronoms personnels et déterminants utilisés } \\
\text { Modes utilisés (indicatif/conditionnel) } \\
\text { « Logique » de l'enchaînement discursif } \\
\text { Comportements prosodiques et co-verbaux : } \\
\text { intonation, débit, gestes... } \\
\text { Dédoublement énonciatif lié à un commentaire } \\
\text { réflexif explicite }\end{array}$ \\
\hline
\end{tabular}

\section{Annexe 2. Conventions de retranscription et codage utilisé dans les extraits de dialogue. Transcription and coding conventions used in the dialogue extracts}

Conventions de retranscription :

: plus ou moins nombreux indiquent un allongement vocalique (pause remplie) et sa durée relative (plus il y a de :, plus elle est longue)

+ plus ou moins nombreux indiquent un silence dans l'énoncé et sa durée relative (plus il y a de + , plus elle est longue)

(indication) : indications portant sur des manifestations sonores ou paraverbales que l'on peut entendre (rires, inspirations sonores...)

(indication) : indications portant sur des manifestations non verbales que l'on peut voir (gestes coverbaux essentiellement)

/ délimite l'abandon d'une construction, soit parce que le locuteur s'interrompt, soit parce qu'il est interrompu (par quelqu'un ou quelque chose) 
[coupure] : coupure dans la retranscription

Les protagonistes, à chaque extrait, sont lors de leur première prise de parole désignés par leur pseudonyme, puis pas l'initiale de leur pseudonyme.

IP correspond à : intervenante psychologue

Codage des principaux indices linguistiques retenus pour l'analyse :

surligné gris : les pronoms, les modalisateurs principaux et les commentaires réflexifs

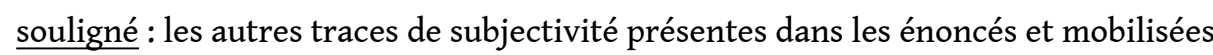
dans l'analyse

en gras : les éléments de contenu portant sur l'objet de l'activité analysée, et sur l'activité réalisée et/ou réellement menée (actions entreprises, mobiles, buts...)

\section{NOTES}

1. Nous utilisons à un premier niveau ce terme générique d'« engagement ", mais nous verrons par la suite que différents qualificatifs ou compléments lui sont accolés, selon les conceptualisations, pour spécifier ce dont on parle.

2. Les chefs d'équipe du nettoiement ont la responsabilité d'un atelier d'une trentaine d'éboueurs. Ils ont pour tâche d'organiser au quotidien le travail des éboueurs et d'en contrôler la réalisation, afin d'assurer la propreté «optimale » d'un secteur géographique. Cela implique, entre autres, un travail de planification et de répartition des tâches, un accompagnement du travail des éboueurs et une présence régulière dans les rues de leur secteur.

3. La commune dans laquelle nous sommes intervenues a divisé son territoire géographique en plusieurs divisions de la propreté, qui elles-mêmes sont redécoupées en secteurs. Dans chaque secteur, plusieurs ateliers prennent chacun en charge la propreté d'une zone de rues.

4. Les citations en italique sont des extraits de propos recueillis en intervention, contrairement aux citations de typographie normale qui sont des extraits d'ouvrages ou d'articles.

5. Lors de cette première intervention, c'est ainsi que les chefs d'équipe formulent leur prise en compte de cette problématique de l'absentéisme en expliquant que «le travail de l'encadrement consiste justement à faire revenir les éboueurs le lendemain ", c'est-à-dire à faire en sorte que leur expérience du jour ne les amène pas à être tentés le lendemain de « se soustraire au travail par la voie de l'absentéisme » (Bonnemain, 2015, p. 63).

6. Nous ne rentrerons pas dans le détail ici, mais dans la méthode des ACS et des ACC, les autoconfrontations se font à partir de films de séquences d'activité. Les activités retenues pour l'analyse ne permettaient que difficilement la réalisation de films d'activité, notamment de par leur nature peu prévisible. Ceci nous a amenées à faire porter les ACS et ACC sur quelques films d'activité, mais aussi sur des documents remplis ou confectionnés par les chefs d'équipe au cours de leur activité, et sur des films descriptifs de l'activité mettant bout à bout des extraits d'ACS dans lesquels un chef d'équipe décrit de façon factuelle (sans explication ou justification) ce qu'il a fait.

7. Les lieux et les noms ont été changés afin de garantir l'anonymat des différents protagonistes de la situation.

8. Elle est alors affectée par la division à une autre zone que la zone prévue dans la programmation hebdomadaire de la division. C'est ce qui s'est passé la veille de cette ACS, comme nous l'apprend l'appel de la stagiaire de la division mentionné plus haut.

9. Un plan de lavage coordonné est un plan qui associe deux ateliers pour nettoyer en même temps une même zone. Dans le cas présent, l'avenue du Layon est un axe limitrophe entre deux 
ateliers. Le nettoyage de son côté pair dépend du premier, celui de son côté impair du second, mais grâce au plan coordonné les deux côtés sont censés être nettoyés le même jour.

\section{RÉSUMÉS}

Cet article se propose de revisiter la question de l'engagement et du désengagement au travail, en étudiant ces derniers dans leurs liens avec l'activité de travail, telle qu'elle est quotidiennement menée par les professionnels en situation. Il mobilise pour ce faire différentes perspectives théoriques, issues de la psychopathologie et des cliniques du travail ; ainsi que des données issues d'une intervention en clinique de l'activité menée dans un service municipal de la propreté. En s'appuyant sur un exemple, il identifie une configuration particulière d'engagement et de désengagement au travail, ses liens avec l'activité, ainsi que ses implications subjectives et ses effets sur le travail.

This article revisits the question of commitment and disengagement at work, by studying their links with the work activity as it is carried out on daily basis by professionals. We mobilize different theoretical perspectives, rooted in psychopathology and in clinical approaches to workplaces and work activity. These perspectives are discussed in relation to data from an intervention carried out in a public cleaning department. This intervention was conducted using the Clinic of Activity approach. Taking one example, we identified a specific configuration of commitment and disengagement at work, its links with the activity, and its subjective implications and effects on daily work.

\section{INDEX}

Keywords : commitment at work, disengagement from work, activity analysis, simple and crossed self-confrontation, clinical approaches to work analysis

Mots-clés : engagement au travail, désengagement au travail, analyse de l'activité, autoconfrontations simples et croisées, cliniques du travail

\section{AUTEURS}

\section{AMÉLIE SANDOVAL}

Centre de Recherche sur le Travail et le Développement (CRTD, EA 4132), 41, rue Gay Lussac, 75005 Paris amelie.sandoval@lecnam.net

\section{KATIA KOSTULSKI}

Centre de Recherche sur le Travail et le Développement (CRTD, EA 4132), 41, rue Gay Lussac, 75005 Paris katia.kostulski@lecnam.net 